EXTENDED REPORT

\title{
Photodynamic therapy with verteporfin for subfoveal choroidal neovascularisation of pathologic myopia in Chinese eyes: a prospective series of 1 and 2 year follow up
}

\author{
D S C Lam, W-M Chan, D T L Liu, D S P Fan, W W Lai, K K L Chong
}

Br J Ophthalmol 2004;88:1315-1319. doi: 10.1136/bjo.2004.041624

See end of article for authors' affiliations

Correspondence to: Dr W-M Chan, Department of Ophthalmology \& Visual Sciences, The Chinese University of Hong Kong, University Eye Center, Hong Kong Eye Hospital, 147K, Argyle Street, Hong Kong; cwm6373@ netvigator.com

Accepted 21 March 2004

\begin{abstract}
Aims: To evaluate the visual and fluorescein angiographic outcomes of photodynamic therapy (PDT) with verteporfin in patients with subfoveal choroidal neovascularisation (CNV) caused by pathologic myopia in the Chinese.

Methods: Prospective, non-comparative, two centre interventional study. Patients with CNV secondary to pathologic myopia of Chinese ethnicity were recruited and treated with a standard regimen of PDT with verteporfin. Results of this study in Chinese eyes with pigmented retinal pigment epithelium were compared with those from the Verteporfin in Photodynamic Therapy (VIP) Study of predominantly white eyes. Results: Thirty one and 22 eyes that completed the 12 month and 24 month follow up studies respectively were analysed. The mean and median best corrected visual acuities (BCVA) could be maintained at the baseline level at the 12 month and 24 month visits. Fourteen (63.6\%) eyes had stable or improved BCVA at 24 months and six (27.3\%) of them had a moderate gain in vision (improved by three or more lines). Visual results were comparable with that of the VIP study, but the average accumulative PDT treatments required in one and two years were 1.7 and 2.3 respectively, which were significantly less than 3.4 and 5.1 treatments in VIP study. Mean logMAR BCVA of the younger age group ( $<55$ years) at 24 months was 0.41 (SD 0.29), which was significantly better than the older age group ( $\geqslant=55$ years) of 0.82 (SD 0.40) (Mann-Whitney $U$ test, $p=0.029$ ).

Conclusions: PDT using the predetermined treatment protocol has achieved similar visual outcomes in the Chinese population as in white people with subfoveal myopic CNV over a 2 year study period. The complete cessation of CNV leakage can be accomplished, on average, with fewer PDT retreatments than reported in the VIP study. The disparity may be due to ethnic differences in these two populations.
\end{abstract}

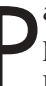
athologic myopia is more common in Asian adult population and the prevalence is around $9 \%^{1}$ which is much higher than that of $2 \%$ in the United States. ${ }^{2}$ High myopia is the seventh leading cause of legal blindness in adult Americans ${ }^{3}$ and ranks second in the blind registration of Hong Kong where the majority of the population is Chinese. ${ }^{4}$ Retinal and macular abnormalities including choroidal neovascularisation (CNV), chorioretinal atrophy, and retinal detachment are some of the ocular complications in patients with high myopia of excessive axial length. ${ }^{5}$ The estimated risk in developing CNV from pathologic myopia is about $4-10 \%^{6-8}$ and the natural course of subfoveal CNV is somewhat controversial but generally regarded as poor. ${ }^{7-12}$ In a series of Asian patients with myopic subfoveal CNV, the best corrected visual acuity (BCVA) dropped by 2.0 lines in a 3 year follow up, 5.0 lines in 5 years, and 5.7 lines in 10 years. ${ }^{13}$ Avila et al observed 70 eyes with untreated CNV from a predominantly white population with an average follow up of 41 months; $51 \%$ have stable or improved visual acuity. ${ }^{9}$

Different modalities of treatment, including direct laser photocoagulation and submacular surgery, have been proposed in the management of subfoveal CNV secondary to pathologic myopia but the outcomes were not encouraging. ${ }^{14-16}$ The one year results of the Verteporfin in Photodynamic Therapy (VIP) trial demonstrated that verteporfin treated patients had a higher chance of stabilising or improving vision in patients with subfoveal $\mathrm{CNV}$ secondary to pathologic myopia. ${ }^{17}$

There is a wide diversity in prevalence of fundus diseases between Asian and white eyes. ${ }^{19-22}$ The different degrees of pigmentation in the retinal pigment epithelial layers of dissimilar races may also affect the optimal treatment parameters and outcomes of treatments through diverse mechanisms. ${ }^{23-26}$ In the VIP study, 91\% of patients were white and only $4 \%$ Asian. ${ }^{17}$ Whether Asian or Chinese eyes with pigmented retinal pigment epithelium layers respond differently to the standard verteporfin infusion protocol and predetermined laser energy level is not known. This prospective study investigated the treatment outcomes of PDT on myopic subfoveal CNV in the Chinese population over one and two year periods.

\section{SUBJECTS AND METHODS}

This was a prospective, consecutive, interventional study involving two centres aimed at investigating the safety and efficacy of PDT with verteporfin for subfoveal CNV of pathologic myopia in the Chinese population. Subfoveal is defined as the involvement of the centre of the foveolar avascular zone. ${ }^{17}$ The protocol was reviewed and approved by the ethics committee of the Chinese University of Hong Kong and consent was obtained from each patient.

\section{Patient selection}

Patients were recruited from two tertiary ophthalmic centres in Hong Kong: Hong Kong Eye Hospital and Prince of Wales Hospital, and enrolment started in February 2000.

Abbreviations: $B C V A$, best corrected visual acuity; $C N V$, choroidal neovascularisation; FA, fluorescein angiography; PDT, photodynamic therapy; RPE, retinal pigment epithelium; VIP Study, Verteporfin in Photodynamic Therapy Study. 
Inclusion criteria of the study included: (1) pathologic myopia defining as high myopia with spherical equivalent $\leqslant-6.0$ dioptres or axial length $\geqslant 26.5 \mathrm{~mm}$; (2) aged 18 years or older; (3) CNV at the subfoveal area; (4) greatest linear dimension of the entire lesion not exceeding $5400 \mu \mathrm{m}$; (5) BCVA of at least 20/200 or better, and (6) active CNV in clinical and in angiography.

Exclusion criteria included: (1) other clinical features suggesting that the CNV may be secondary to causes other than pathologic myopia (such as large drusen, geographical atrophy, choroioretinal scar); (2) any systemic contraindications for verteporfin or angiographic dyes; (3) patients who had received previous treatment before enrolment; (4) significant concurrent ocular or macular diseases.

Pretreatment assessment and photodynamic therapy All participants received a comprehensive ocular examination including dilated fundus and macular examinations with a contact lens or a 90 diopter lens, followed by colour fundus photography, fluorescein angiography (FA) at baseline and then every 3 months. BCVA was measured by certified optometrist with a standard Snellen chart. In order to quantify differences in vision, all Snellen BCVA have been converted to the logarithm of the minimum angle of resolution (logMAR) BCVA. ${ }^{27}$ Photodynamic therapy was given with the standard verteporfin (Visudyne, Novartis AG, Bülach, Switzerland) infusion followed by laser application at $689 \mathrm{~nm}$ (Coherent Inc, CA, USA). ${ }^{17}$

\section{Follow up, retreatment, and end point}

Patients were seen every 3 months and retreatment was considered if there was persistent or new leakage in FA. Retreatment was given in the same manner as the initial treatment described. Treatment end point was deemed achieved when there was complete absence of angiographic leakage in FA. In equivocal cases, a biomicroscropic contact lens examination together with optical coherence tomography in delineating the morphology of fibrotic CNV and absence of subretinal or intraretinal fluid were helpful.

\section{Outcome measurements}

Primary outcomes were proportion of eyes that had improved (one or more lines), stable, or decreased (one or more lines) vision at the end of the first and second years. Mean and median changes in visual acuities at months 12 and 24 were measured.

Secondary outcome parameters included number of treatments required, the associated factors for better outcomes, and proportion of eyes with final vision loss of three or more lines. Any ocular or systemic adverse events caused by PDT were also tabulated.

\section{Statistical analysis}

Demographic characteristics of patients including the angiographic findings were summarised by descriptive statistics using statistical software (SPSS version 10.1.0, SPSS Inc, Chicago, IL, USA). Comparisons between initial and final visual acuities and among different groups were performed by Wilcoxon signed ranks test and Mann-Whitney $U$ test respectively. Potential risk factors such as age and degree of myopia were analysed by Pearson correlation.

\section{RESULTS}

\section{Characteristics of patients}

From February 2000 to September 2003, 31 eyes from 30 patients with subfoveal CNV secondary to pathologic myopia had completed 1 year's follow up and of them, 22 eyes from 21 patients had completed 2 years' follow up. There were 20 $(66.7 \%)$ females and $10(33.3 \%)$ males; all were southern
Chinese. Seventeen patients involved the left eye whereas 12 involved the right and one had bilateral involvement. Mean (standard deviation (SD)) of age at presentation was 51.6 (SD 14.8) years, range 31-82 years. Refractive error ranged from $-6.00 \mathrm{D}$ to $-22.00 \mathrm{D}$ and mean $(\mathrm{SD})$ was -10.65 (SD 4.13) D.

Visual outcomes at 1 year of follow up ( 31 eyes)

On initial presentation, the mean logMAR BCVA was 0.61 (SD 0.32). The initial Snellen BCVA ranged from 20/25 to 20/ 200 with a mean of $20 / 81$. The median Snellen BCVA on presentation was 20/100 (logMAR 0.70). At 1 year of follow up, the mean logMAR BCVA was 0.57 (SD 0.36). The Snellen BCVA ranged from $20 / 20$ to $20 / 200$ with a mean of 20/74. The median Snellen BCVA was 20/70 (logMAR 0.54).

Thirteen $(41.9 \%)$ eyes had BCVA improved by one or more lines and eight $(25.8 \%)$ of these eyes had a moderate gain in vision (three or more lines). Twelve (38.7\%) eyes had stable vision and six (19.4\%) eyes had visual loss of one line or more. The mean and median lines of improvement were 0.7 and 1.6 lines respectively, but the change in the mean logMAR BCVA at 12 months was not statistically significant (Wilcoxon signed ranks test, $\mathrm{p}=0.145$ ). The changes in BCVA of each individual case at 12 months are displayed in the scatter diagram (fig 1). At baseline, nine (29.0\%) patients had initial BCVA of 20/40 or better. At the 12 month follow up, $12(38.7 \%)$ patients had BCVA of 20/40 or better. This, however, was not statistically significant (McNemar test, $\mathrm{p}=0.508$ ).

\section{Visual outcomes at 2 years' follow up (22 eyes)}

For those eyes that completed the 2 years' follow up, initial mean logMAR BCVA was 0.59 (SD 0.34). At 2 years' follow up, the mean logMAR BCVA was 0.56 (SD 0.38). There was also no statistically significant change in the mean BCVA (Wilcoxon signed ranks test, $\mathrm{p}=0.500$ ). The initial and the final median $\log$ MAR BCVA were 0.70 and 0.47 respectively. Most of the visual improvement occurred within the first 3 months after the initial PDT, but the visual gain was gradually lost and the vision returned back to the baseline level in subsequent follow ups (fig 2).

Eight $(36.4 \%)$ eyes had final BCVA improved by one or more lines and in which six $(27.3 \%)$ of these eyes had a moderate gain in vision (three or more lines). Eight (36.4\%) eyes had visual loss of one line or more and remaining six

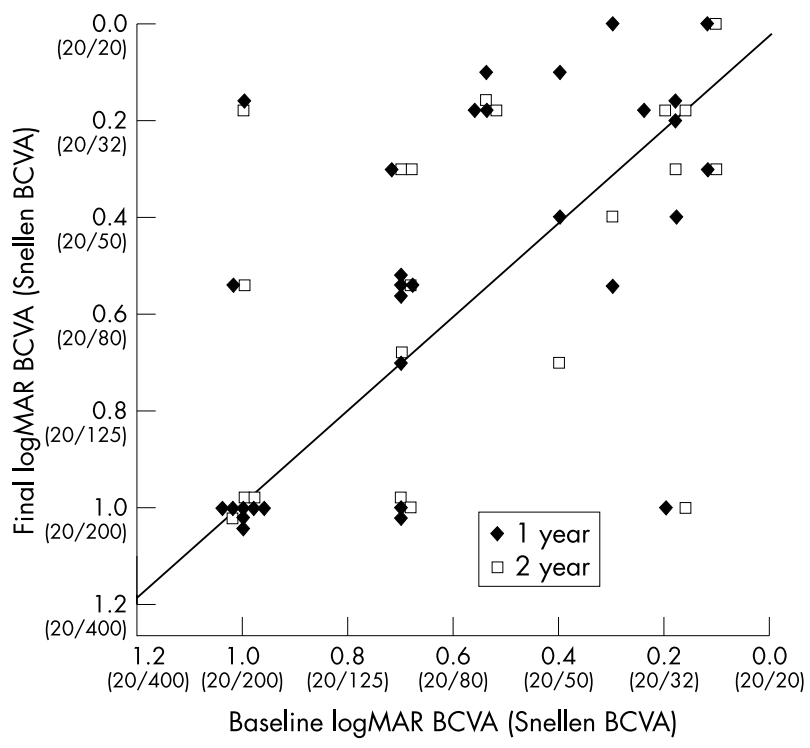

Figure 1 Scatter plot showing the change in visual acuities at one and two year visits with photodynamic therapy 


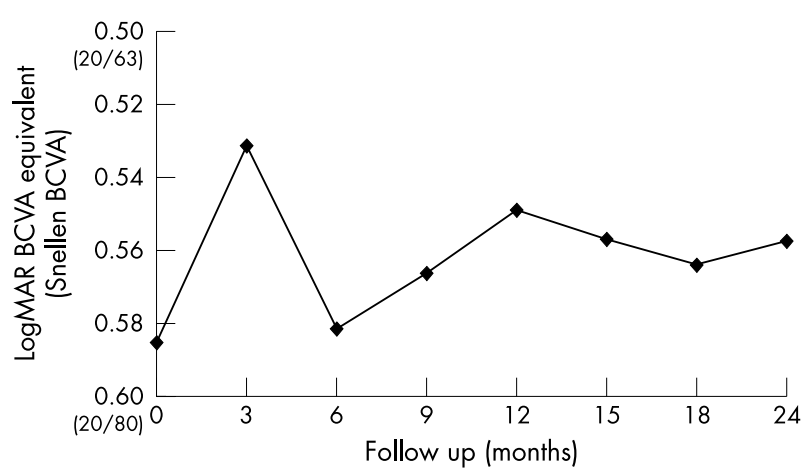

Figure 2 Changes in the mean logMAR BCVA with time over the 24 month follow up

$(27.3 \%)$ eyes had stable vision. Changes in BCVA of each individual case at 24 months are displayed in the scatter diagram (fig 1).

\section{Number of PDT sessions}

A total of 54 treatment sessions had been given to 31 eyes in the first year with a mean of 1.7 treatments. Eighteen (58.0\%) eyes received only one single PDT session during the 12 month study period. Retreatment was performed for cases with persistent, recurrent or new angiographic leakage in FA. Four $(12.9 \%)$ eyes required two PDT sessions, eight $(25.8 \%)$ eyes required three, and one (3.2\%) eye required four sessions of PDT.

In the second year, most of the eyes remained stable angiographically. One had persistent CNV that required an additional three more sessions in the second year. Four had recurrent $\mathrm{CNV}$ in the second year, in which two received one more and the other two received two more sessions. On average, the cumulative treatment sessions needed for the 22 study eyes over the 2 year follow up period was 2.3 (figs 3 and 4).

\section{Risk factor analysis}

Age is not related to the preoperative VA $(r=0.321$, $\mathrm{p}=0.079)$. However, the younger the patient, the better the VA at 12 months $(r=0.594, \mathrm{p}<0.001)$ and 24 months $(r=0.501, \mathrm{p}=0.018)$ after PDT. In addition, younger patients also have better improvement in visual acuity at 12 months $(r=0.386, \mathrm{p}=0.032)$ and 24 months $(r=0.445, \mathrm{p}=0.038)$ postoperatively.

In further analysis with different age groups $1<55$ and $\geqslant 55$ years), the mean logMAR VA for patients $<55$ years was 0.36 (SD 0.28) and that for patients $\geqslant 55$ years was 0.82 (SD 0.32 ) (Mann-Whitney $U$ test, $p=0.001$ ). The mean logMAR $\mathrm{VA}$ at 24 months also showed that patients in the younger age group ( $<55$ years) (0.41 (SD 0.29)) were significantly better than patients in the older age group ( $\geqslant 55$ years) (0.82 (SD 0.40)) (Mann-Whitney U test, $\mathrm{p}=0.029)$. The median logMAR VA at 12 months for patients $<55$ years and $\geqslant 55$ years were 0.30 and 1.00 respectively; the median $\log$ MAR VA at 24 months for patients $<55$ years and $\geqslant 55$ years were also 0.30 and 1.00 respectively.

Concerning different degrees of refractive errors $(\geqslant-12 \mathrm{D}$ or $<-12 \mathrm{D}$ ), there is no statistically significant difference in the logMAR VA at 12 months (Mann-Whitney $U$ test, $\mathrm{p}=0.716$ ) and at 24 months (Mann-Whitney $\mathrm{U}$ test, $\mathrm{p}=0.541)$.

\section{Adverse events and complications}

Four developed haemorrhagic complications during the study period. All resolved gradually with time. Transient visual disturbance was reported in five eyes. Potential but serious complications such as severe acute loss of vision, and rip or tear in RPE did not occur in our series. None of the patients suffered from any discomfort or complications arising from infusion or laser application such as low back pain, catheter induced complications, photosensitivity, and so on.

\section{DISCUSSION}

Choroidal neovascularisation in the subfoveal area can cause significant loss of vision by affecting the RPE and neurosensory retina. ${ }^{78}$ Pathologic myopia is a common underlying disease in causing CNV. Compared with age related macular degeneration, it affects a younger age group and the loss of vision can be severe and may not be recovered with any further treatment.

Hotchkiss et al reported a series of 23 eyes in 23 patients with CNV associated with high myopia. ${ }^{7}$ Deterioration of visual acuity was shown in $51 \%$ of eyes, with a final visual acuity of 20/200 or less in $44 \%$ of eyes. ${ }^{7}$ Avila et al reported a series of 70 eyes in 58 patients with untreated CNV associated with high myopia in which $60 \%$ had the final vision of $20 / 200$ or less. ${ }^{9}$ From the placebo arm of the VIP study, which was the only prospective series in the literatures, 17 out of 39 eyes $(43 \%)$ had stable or improved vision at both 1 year and 2 year follow up. ${ }^{17} 18$

The data gathered so far from submacular surgery for subfoveal CNV of pathologic myopia are less promising, as the visual results are frequently unpredictable and severe early loss of vision is possible. ${ }^{15}{ }^{16}$ Macular translocation surgery may also be considered as a treatment option for subfoveal $\mathrm{CNV}$ associated with pathologic myopia. ${ }^{28}$ However, the surgical techniques are demanding and complications are not uncommon. ${ }^{29}$ Similar to submacular surgery, there has to date been no randomised study demonstrating its long term efficacy.

Some beneficial outcomes have been shown in verteporfin treated group of VIP study. ${ }^{17}$ Primary outcome, however, was no longer statistically significant in the second year follow up study. ${ }^{18}$ That may be caused by the loss of efficacy of PDT or subtle adverse effects of the treatment on neurosensory retina and RPE in long run.

In our study, the vision was preserved at the end of the follow up. No significant functional changes of the mean and median visual acuities at months 12 and 24 from the baseline level were found. The overall visual outcomes are comparable with the VIP study. Indeed, more eyes (63.6\%, 14 of 22 eyes) achieved stable or improved vision in the present series at the completed second year of follow up in comparison with the control group (44\%, 17 of 30 eyes) of the VIP study.

Age is a determinant affecting the outcomes of myopic CNV. Yoshida et al showed that the younger ( $\leqslant 40$ years) generally have a better prognosis. About half the patients retained a final visual acuity better than 20/40 and there was no significant change in mean VA with a follow up period of 3 years or more. Those findings might be accounted for by the smaller size of CNV, and less chorioretinal atrophy in the younger age group. As suggested by the author, the age at the time of onset of myopic CNV should be considered in deciding any intervention. ${ }^{30}$ In the risk factors analyses of our study, it has been shown that younger patients ( $<55$ years) with myopic subfoveal CNV responded better to PDT than the older age group ( $\geqslant 55$ years) at both 12 months and 24 months (Mann-Whitney $\mathrm{U}$ test, $\mathrm{p}=0.001$ and $\mathrm{p}=0.029$ respectively). ${ }^{31}$

The present study adopted the same angiographic endpoint as in the VIP study. The average number of treatments achieving complete absence of leakage in the first year and the accumulated number in the second year was 1.7 and 2.3, which were much lower than 3.4 and 5.1 treatment sessions 


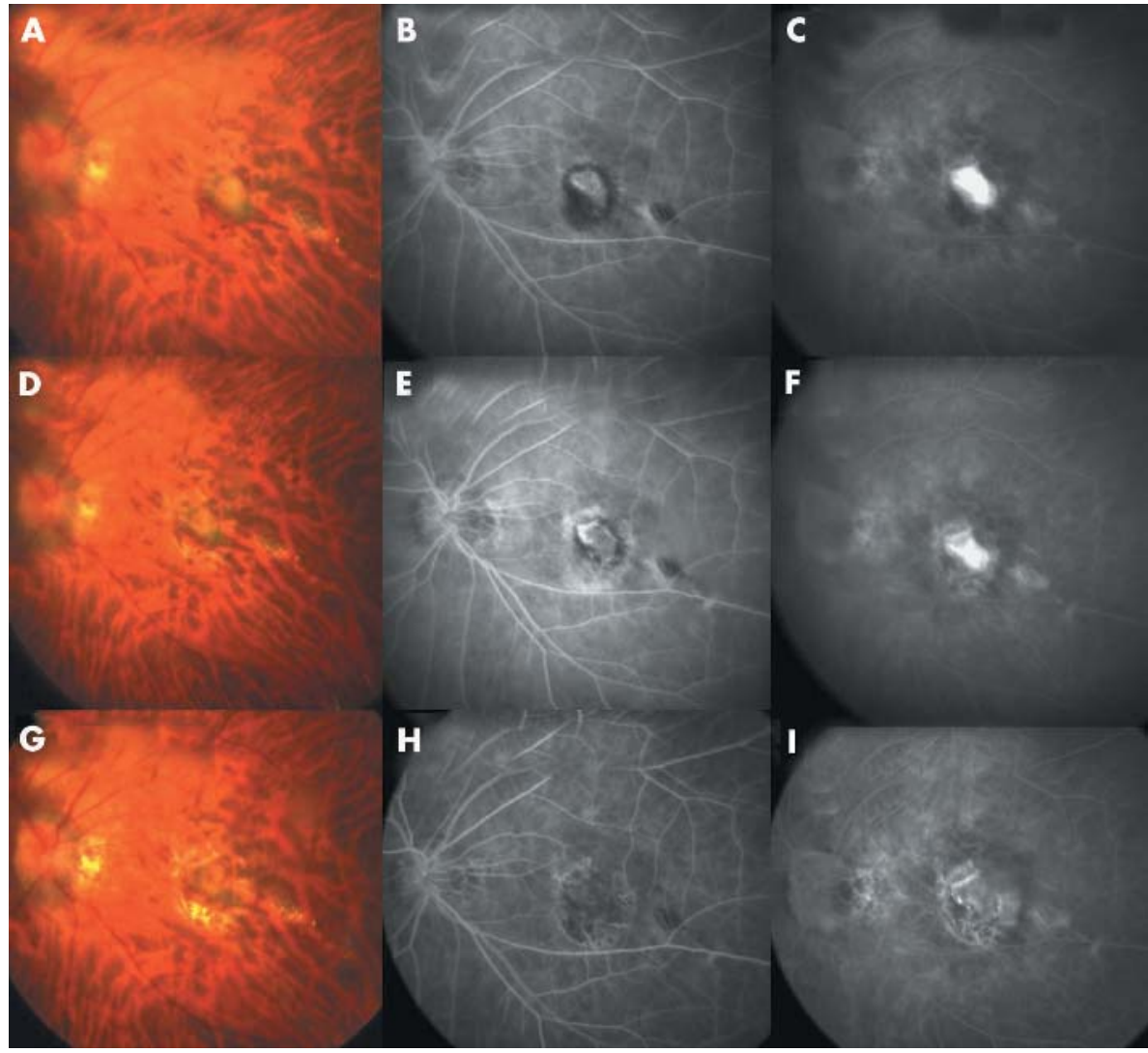

Figure 3 Left eye of a 56 year old Chinese woman with myopia of -8.00 diopters (D) manifesting as choroidal neovascularisation (CNV). The baseline best corrected visual acuity (BCVA) was $20 / 200$. (A) Fundus photography showing CNV of 1 disc diameter at subfoveal region together with a rim of subretinal haemorrhage. (B, C) Early phase fluorescein angiography (FA) showing a well delineated classic membrane with early hyperfluorescence and profuse angiographic leakage in the late phase. (D) Fundus photography at 3 months after photodynamic therapy (PDT) showing a CNV of the same size. The adjacent haemorrhage was partially reabsorbed. $(E, F)$ Early and late phase of FA showing a CNV with mild angiographic leakage with time as shown by increasing

hyperfluorescence, an ill defined border, and increasing in size of hyperfluorescence in late phase compared with early phase. Retreatment with PDT was required in accordance with angiographic manifestations. (G) The CNV becoming a fibrotic scar and associated with atrophy of adjacent retinal pigment epithelium. (H, I) No vasacular leakage was revealed in subsequent follow ups and the early and late phase of the FA at 2 years showed a non-leaking scar. The BCVA remained at $20 / 200$.

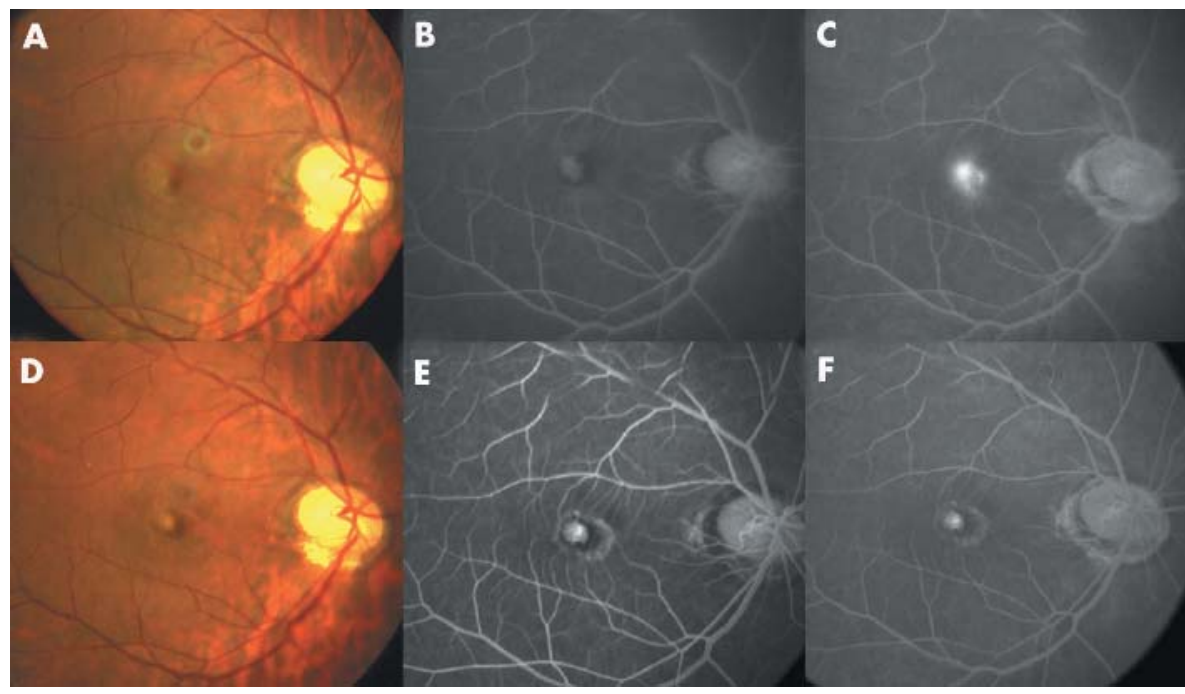

Figure 4 Right eye of a 44 year old Chinese man with myopia of -11.00 D. (A) Fundus photograph revealing a small subfoveal lesion with subretinal haemorrhage. The baseline best corrected visual acuity (BCVA) was 20/100. (B) Early phase fluorescein angiography (FA) showing a tiny well defined choroidal neovascularisation (CNV). (C) Late phase FA showing profuse angiographic leakages from the CNV. (D) Fundus photograph at 3 months after PDT, revealing a small contracting lesion together with a rim of hyperplastic retinal pigment epithelium. (E) Early phase FA showing a circumscribed lesion with hyperfluorescence. (F) Late phase FA showing same level and same dimension of hyperfluorescence. The edge of the lesion remained sharp and clear and it was compatible with scar staining. No repeated PDT was offered and the angiographic morphology remained the same in the subsequent follow ups together with a stable BCVA at the level of $20 / 70$ at the 2 year visit. in the VIP study respectively. The pigmented RPE may provide a more protective effect from the unrestrained growth of the CNV after PDT, or there might be some other confounding factors accounting for the disparity in the average number of PDT treatments. Concerning complications or side effects from the therapy, there are no new safety concerns with the same treatment protocol and laser energy.

Our series, however, has its own limitations including a small sample size, a short follow up period, and no comparative control group. This study design does not allow us to conclude the efficacy of PDT in the Asian population to treat subfoveal CNV from myopic degeneration as there was no control observation arm. The number of retreatments required in achieving stable angiographic results is less in our series. Whether or not the Asian population, which has more fundus pigmentation, responds differently from non-Chinese patients may warrant a comparative trial with controls.

\section{ACKNOWLEDGEMENT}

Supported by Competitive Earmarked Research Grant \#4140/02M. 


\section{Authors' affiliations}

D S C Lam, W-M Chan, D S P Fan, W W Lai, Department of Ophthalmology \& Visual Sciences, The Chinese University of Hong Kong, Hong Kong Eye Hospital, Kowloon, Hong Kong, People's

Republic of China

D S C Lam, W-M Chan, D T L Liv, W W Lai, K K L Chong, Department of Ophthalmology \& Visual Sciences, The Chinese University of Hong Kong, Prince of Wales Hospital, Shatin, NT, Hong Kong, People's Republic of China

Financial interest: none.

\section{REFERENCES}

1 Wong TY, Foster PJ, Hee J, et al. Prevalence and risk factors for refractive errors in adult Chinese in Singapore. Invest Ophthalmol Vis Sci 2000;41:2486-94.

2 Sperduto RD, Seigel D, Roberts J, et al. Prevalence of myopia in the United States. Arch Ophthalmol 1983;101:405-7.

3 Curtin B. The myopias: basic science and clinical management. Philadelphia: Harper and Row, 1985:7-10.

4 Registration of Permanent Blindness in Hospital Authority Ophthalmology: Statistical Report: http://www.ha.org.hk/hesd/nsapi/?Mlval = ha visitor index\&intro = ha\% 5 fview $\% 5 f t e m p l a t e \% 26$ group $\% 3 \mathrm{dIFN} \% 26 \mathrm{Area} \% 3 \mathrm{dPBL}$ (accessed 7 July 2004).

5 Curtin BJ. Physiologic vs pathologic myopia: genetics vs environment Ophthalmology 1979:86:681-91.

6 Curtin BJ, Karlin DB. Axial length measurements and fundus changes of the myopic eye. Am J Ophthalmol 1971;1:42-53

7 Hotchkiss ML, Fine SL. Pathologic myopia and choroidal neovascularization Am J Ophthalmol 1981;91:177-83.

8 Grossniklaus HE, Green WR. Pathologic findings in pathologic myopia. Retina 1992;12:127-33.

9 Avila MP, Weiter JJ, Jalkh AE, et al. Natural history of choroidal neovascularization in degenerative myopia. Ophthalmology 1984;91:1573-81.

10 Hampton GR, Kohen D, Bird AC. Visual prognosis of disciform degeneration in myopia. Ophthalmology 1983;90:923-6.

11 Adelberg DA, Del Priore LV, Kaplan HJ. Surgery for subfoveal membranes in myopia, angioid streaks, and other disorders. Retina 1995;15:198-205.

12 Tabandeh H, Flynn HW Jr, Scott IU, et al. Visual acuity outcomes of patients 50 years of age and older with high myopia and untreated choroidal neovascularization Ophthalmology 1999;106:2063-7.

13 Yoshida T, Ohno-Matsui K, Yasuzumi K, et al. Myopic choroidal neovascularization: a 10-year follow-up. Ophthalmology 2003:1 10:1297-305.

14 Jalkh AE, Weiter JJ, Trempe CL, et al. Choroidal neovascularization in degenerative myopia: role of laser photocoagulation. Ophthalmic Surg 1987; 18:721-5.
15 Thomas MA, Dickinson JD, Melberg NS, et al. Visual results after surgical removal of subfoveal choroidal neovascular membranes. Ophthalmology 1994; 101:1384-96.

16 Ruiz-Moreno JM, de La Vega C. Surgical removal of subfoveal choroidal neovascularisation in highly myopic patients. $\mathrm{Br} J \mathrm{O}$ Ophthalmol 2001;85:1041-3.

17 Verteporfin in Photodynamic Therapy Study Group. Photodynamic therapy of subfoveal choroidal neovascularization in pathologic myopia with verteporfin. 1-year results of a randomized clinical trial-VIP report no. 1. Ophthalmology 2001;108:841-52.

18 Blinder KJ, Blumenkranz MS, Bressler NM, et al. Verteporfin therapy of subfoveal choroidal neovascularization in pathologic myopia: 2-year results of a randomized clinical trial-VIP report no. 3. Ophthalmology 2003; 1 10:667-73.

19 Vajdic CM, Kricker A, Giblin M, et al. Eye color and cutaneous naevi predict risk of ocular melanoma in Australia. Int J Cancer 2001;92:906-12.

20 Kuo PK, Puliafito CA, Wang KM, et al. Uveal melanoma in China. Int Ophthalmol Clin 1982;22:57-71.

21 Lau JT, Lee V, Fan D, et al. Knowledge about cataract, glaucoma, and age related macular degeneration in the Hong Kong Chinese population. Br J Ophthalmol 2002;86:1080-4.

22 Baum L, Chan WM, Li WY, et al. ABCA4 sequence variants in Chinese patients with age-related macular degeneration or Stargardt's disease. Ophthalmologica 2003;217:111-14.

23 Roider J. Laser treatment of retinal diseases by subthreshold laser effects. Semin Ophthalmol 1999;14:19-26.

24 Auer C, Tao Tran V, Herbort CP. Transpupillary thermotherapy for occult subfoveal neovessels in age-related macular degeneration: importance of patient pigmentation for the determination of laser settings. Klin Monatsb Augenheilkd 2002;219:250-3.

25 Peyman GA, Genaidy M, Moshfeghi DM, et al. Transpupillary thermotherapy threshold parameters: funduscopic, angiographic, and histologic findings in pigmented and nonpigmented rabbits. Retina 2003;23:371-7.

26 Wachtlin J, Behme T, Heimann $\mathrm{H}$, et al. Concentric retinal pigment epithelium atrophy after a single photodynamic therapy. Graefes Arch Clin Exp Ophthalmol 2003;241:518-21.

27 Holladay JT. Proper method for calculating average visual acuity. J Refract Surg 1997;13:388-91.

28 Fujikado T, Ohji M, Saito Y, et al. Visual function after foveal translocation with scleral shortening in patients with myopic neovascular maculopathy. Am J Ophthalmol 1998; 125:647-56.

29 Sawa M Chan WM, Ohii M, et al. Successful photodynamic therapy with verteporfin for recurrent choroidal neovascularization beneath the new fovea after macular translocation surgery with 360-degree retinotomy. Am J Ophthalmol 2003;136:560-3.

30 Yoshida T, Ohno-Matsui K, Ohtake Y, et al. Long-term visual prognosis of choroidal neovascularization in high myopia: a comparison between age groups. Ophthalmology 2002;109:712-19.

31 Montero JA, Ruiz-Moreno JM. Verteporfin photodynamic therapy in highly myopic subfoveal choroidal neovascularisation. Br J Ophthalmol 2003:87:173-6. 